\title{
Cómo realizar e interpretar una manometría esofágica de alta resolución usando la clasificación de Chicago 3.0
}

\section{How to Perform High Resolution Esophageal Manometry and How to Interpret It Using Chicago 3.0}

Albis Hani, ${ }^{1}$ Walter Bernal, ${ }^{2}$ Ana María Leguízamo, ${ }^{1}$ Claudia Zuluaga, ${ }^{1}$ Rómulo Vargas, ${ }^{1}$ Hernando Vergara, ${ }^{1}$ Andrés Ardila Hani, ${ }^{1}$ Valeria Costa. ${ }^{1}$

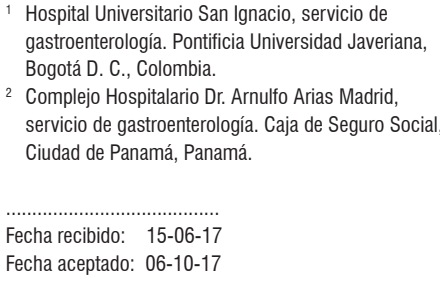
gastroenterología. Pontificia Universidad Javeriana, Bogotá D. C., Colombia.

2 Complejo Hospitalario Dr. Arnulfo Arias Madrid, servicio de gastroenterología. Caja de Seguro Social, Ciudad de Panamá, Panamá.

Fecha recibido: $15-06-17$

Fecha aceptado: 06-10-17

\begin{abstract}
Resumen
Con la introducción de la manometría esofágica de alta resolución se revelaron patrones no identificados previamente de la función esofágica. De igual forma, este método diagnóstico adiciona patrones de presión topográfica de la presión esofágica, lo que lleva al desarrollo de nuevas herramientas para el análisis y clasificación de desórdenes motores esofágicos. En la actualidad, la clasificación de Chicago 3.0 es la herramienta de análisis de los diferentes trastornos motores esofágicos. En Colombia, cada día se ve el crecimiento en la realización de este estudio. El artículo propone hacer una revisión de cómo realizar e interpretar una manometría esofágica de alta resolución y clasificar los diferentes trastornos de la motilidad esofágica según la última actualización de la clasificación de Chicago 3.0.
\end{abstract}

Palabras clave

Desórdenes de motilidad esofágica, manometría esofágica de alta resolución, acalasia, Chicago 3.0.

\begin{abstract}
The introduction of high resolution esophageal manometry has led to the revelation of previously unidentified patterns of esophageal function. Similarly, this diagnostic method has revealed topographic patterns of esophageal pressure which has led to the development of new tools for analysis and classification of esophageal motility disorders. Currently, the Chicago 3.0 classification has become a tool for analysis of the various esophageal motility disorders. In Colombia, the use of this study is spreading and growing. This article reviews of how to perform and interpret high resolution esophageal manometry and shows how to classify esophageal motility disorders according to the latest update of Chicago 3.0.
\end{abstract}

Keywords

Esophageal motility disorders, high resolution esophageal manometry, achalasia, Chicago 3.0.

\section{INTRODUCCIÓN}

La evaluación de la función motora esofágica y gastrointestinal con técnicas manométricas llegó a ser posible en la década de 1970 cuando Wyle Jerry Dodd y Ron Arndorfer desarrollaron el primer sistema de manometría. En la década de 1990, Ray Clouse y sus colegas dieron nacimiento a la manometría de alta resolución (MAR) al disminuir el espacio entre los sensores ubicados a lo largo del catéter de presión de manometría convencional de $5 \mathrm{~cm}$ a $1 \mathrm{~cm}$ y aumentó el número de sensores y la longitud del segmento sensado desde la faringe hasta el estómago, por lo que logró observar la función motora del esfínter esofágico superior (EES) y el esfínter esofágico inferior (EEI) con 
cada deglución, lo que ofrece una descripción temporoespacial completa de la función motora esofágica $(1,2)$.

Los primeros intentos de clasificar los desórdenes de motilidad esofágica fueron realizados por Spechler y Castell en 2001 con base en una revisión de los datos de la literatura hasta ese momento, utilizando criterios de manometría convencional. Sin embargo, los investigadores reconocieron que el significado clínico de cualquier hallazgo manométrico observado podría estar limitado debido a que muchas de las anormalidades reportadas tenían pobre correlación con los síntomas y el manejo de éstas no llevaba a mejoría de los mismos (3).

El desarrollo de los catéteres de alta resolución y de un software que realiza los trazados con códigos de color y segmentos de presión ha revolucionado el método de evaluación diagnóstica de los trastornos motores esofágicos, con grandes ventajas al comparar los resultados con los obtenidos por estudios de manometría convencional $(4,5)$. Esto llevó en pocos años a que la MAR esofágica y la topografía de presión esofágica (EPT) comenzaran a ser utilizadas en el escenario de investigación clínica y la práctica clínica. El uso incrementado de la MAR permitió una evaluación objetiva de las medidas de EPT que se integraron en un nuevo esquema de clasificación de trastornos motores esofágicos, referida como la clasificación de Chicago, la cual, luego de su primera publicación en 2009 , ha sido intermitentemente actualizada en un intento de representar los fenotipos clínicamente relevantes (3, 6). Después de su última actualización en 2014 (la clasificación de Chicago 3.0), se han publicado varios estudios demostrando que este nuevo esquema resulta en una proporción incrementada de estudios clasificados como normales por disminuir el diagnóstico de trastornos menores de la motilidad esofágica, mientras que la proporción de trastornos mayores de motilidad y trastornos obstructivos permanece sin cambios $(7,8)$.

\section{INDICACIONES}

La manometría esofágica es la prueba definitiva para evaluar la motilidad esofágica y está indicada en la evaluación diagnóstica de pacientes con disfagia no obstructiva cuando los estudios endoscópicos y radiológicos no han aclarado la etiología de la misma, en la evaluación preoperatoria de pacientes antes de la cirugía antirreflujo, en la evaluación del dolor torácico no cardíaco y en la evaluación de individuos sintomáticos después de la cirugía antirreflujo (Tabla 1). Por tanto, es un reto que los gastroenterólogos y los fellows en gastroenterología estén suficientemente familiarizados con la MAR para su uso apropiado en la práctica clínica, con el fin de lograr un manejo óptimo de los pacien- tes con desórdenes primarios o secundarios de la motilidad $(4,9,10)$.

\section{EL LABORATORIO DE MOTILIDAD}

Hacer un adecuado diagnóstico de un trastorno de motilidad esofágica depende en gran medida de obtener un estudio de MAR de alta calidad y esto tiene gran importancia porque del resultado depende el manejo quirúrgico, médico o endoscópico que se le ofrece al paciente. Un laboratorio de manometría esofágica de alta calidad requiere del equipo apropiado y un personal dedicado a desarrollar estudios de MAR (9).

En la actualidad se pueden encontrar equipos fabricados por diferentes casas comerciales como el modelo Sandhill (Sandhill Scientific Inc., Highland Ranch, Colorado, Estados Unidos) y el modelo de Medtronic (Given Imaging, Los Ángeles, California, Estados Unidos), entre otros (11); este último se utilizó en todos los trabajos de investigación para el desarrollo de la clasificación de Chicago (9). El catéter de MAR es de estado sólido (considerado tan económico como un sistema de perfusión y requiere menos mantenimiento) y tiene un diámetro externo de $4,2 \mathrm{~mm}$, con 36 sensores circunferenciales separados en intervalos de $1 \mathrm{~cm}$ $(4,11)$ que permite evaluar la presión intraluminal a través de toda la extensión del esófago y en los esfínteres (1). Un aspecto importante es dar el mantenimiento adecuado a los equipos por personal capacitado y programar el reemplazo oportuno de los catéteres.

Los estudios de manometría esofágica pueden ser realizados por personal médico, de enfermería o técnicos capacitados en él área, que deben contar con el adecuado nivel de entrenamiento y experiencia requeridos para realizar estudios de alta calidad. Este personal debe tener la habilidad de evaluar si el estudio es apropiado, tener suficiente conocimiento para determinar la posición apropiada transdiafragmática del catéter, reconocer artefactos comunes y el mal funcionamiento del equipo (9).

\section{CÓMO REALIZAR UN ESTUDIO DE ALTA CALIDAD}

Desde que la MAR esofágica se introdujo en la práctica clínica, se han desarrollado cambios técnicos y metodológicos, por esto se requiere una estandarización, establecimiento de valores de referencia y criterios de análisis entre los laboratorios (12). Realizar un estudio de MAR de alta calidad requiere controlar ciertos aspectos relacionados con el paciente y la ejecución técnica del procedimiento, como se describe a continuación.

Idealmente, se debe indicar al paciente mantener un ayuno de 6 horas para sólidos y al menos de 2 horas para 
Tabla 1. Recomendaciones para el uso clínico de manometría esofágica

\begin{tabular}{|c|c|}
\hline Disfagia & $\begin{array}{l}\text { Establecer el diagnóstico de desórdenes mayores de motilidad (p. ej.: acalasia, DES). } \\
\text { Soportar el diagnóstico de disfagia funcional luego de excluir la motilidad esofágica anormal. } \\
\text { Identificar una afección esofágica en casos de enfermedad sistémica. }\end{array}$ \\
\hline $\begin{array}{l}\text { Dolor torácico no } \\
\text { cardíaco }\end{array}$ & $\begin{array}{l}\text { Establecer el diagnóstico de desórdenes mayores de motilidad esofágica (p. ej.: trastornos hipercontráctiles). } \\
\text { Soportar el diagnóstico de dolor torácico funcional luego de excluir una motilidad esofágica anormal. } \\
\text { Identificar una afección esofágica de una enfermedad sistémica. }\end{array}$ \\
\hline $\begin{array}{l}\text { Síntomas de } \\
\text { reflujo (pirosis/ } \\
\text { regurgitación) }\end{array}$ & $\begin{array}{l}\text { Antes de la monitorización de reflujo para la colocación de dispositivos intraluminales (p. ej.: catéteres de pH, impedancia). } \\
\text { Antes de la cirugía antirreflujo para excluir/identificar desórdenes mayores de motilidad (p. ej.: acalasia). En postquirúrgico } \\
\text { en pacientes sintomáticos. } \\
\text { Soportar el diagnóstico de síndrome de rumiación en ERGE refractaria. }\end{array}$ \\
\hline Acalasia & $\begin{array}{l}\text { Establecer el mejor abordaje terapéutico basado en los patrones manométricos (p. ej.: dilatación neumática para acalasia } \\
\text { tipos I y II, miotomía de Heller/POEM para todo tipo de acalasia). } \\
\text { En el posquirúrgico en pacientes sintomáticos. }\end{array}$ \\
\hline $\begin{array}{l}\text { Potenciales } \\
\text { indicaciones }\end{array}$ & $\begin{array}{l}\text { Antes de la cirugía bariátrica para excluir/identificar desórdenes mayores de motilidad ( } \mathrm{p} . \text { ej.: acalasia). } \\
\text { Antes de la cirugía antirreflujo para seleccionar la intervención quirúrgica más apropiada (funduplicatura Toupet en caso de } \\
\text { motilidad esofágica inefectiva con múltiples degluciones rápidas anormales). } \\
\text { Evaluar la presencia y tamaño de hernia hiatal para considerar su impacto sobre síntomas potencialmente relacionados con } \\
\text { el reflujo. }\end{array}$ \\
\hline Contraindicaciones & $\begin{array}{l}\text { Absolutas: } \\
\text { Obstrucción esofágica de un proceso infiltrativo (p. ej.: tumor). } \\
\text { Anormalidad nasal u orofaríngea que limita la inserción del catéter. } \\
\text { Franca aspiración con la deglución de líquidos (se pueden realizar tragos secos). } \\
\text { Parámetros de coagulación significativamente alterados. } \\
\text { Relativas: } \\
\text { Pacientes con anticoagulación crónica. } \\
\text { Inhabilidad para tolerar el catéter. }\end{array}$ \\
\hline
\end{tabular}

Adaptado de: Savarino E, et al. Dig Liver Dis. 2016;48(10):1124-35. DES: espasmo esofágico distal; ERGE: enfermedad por reflujo gastroesofágico; POEM: miotomía endoscópica transoral.

líquidos antes del procedimiento. En el caso de sospecha o diagnóstico previo de acalasia, el tiempo de ayuno debe ser de al menos 12 horas; esto disminuye el riesgo de vómito y, por ende, de broncoaspiración durante la introducción del catéter de manometría. Los medicamentos que pueden influenciar la motilidad esofágica deben ser cuidadosamente evaluados $y$, si es posible, se deben descontinuar al menos 3 días antes de la prueba (p. ej.: bloqueadores de canales de calcio, nitratos, procinéticos, loperamida, bloqueantes $\beta$, opiáceos, anticolinérgicos, entre otros) $(1,4,10)$.

$\mathrm{Al}$ tratarse de un procedimiento mínimamente invasivo, se debe suministrar al paciente una detallada explicación acerca de la técnica aplicada en el procedimiento y las posibles incomodidades que va a experimentar. Una vez el paciente haya aprobado la realización del procedimiento, se sugiere realizar la firma del consentimiento informado $(1,9)$.

Previo a la realización de la intubación esofágica del paciente, debe realizarse una calibración automatizada del catéter de manometría, la cual varía de acuerdo con el tipo de sonda que se va a utilizar y las especificaciones del fabricante (1).
El catéter de MAR se coloca vía transnasal, se posiciona observando la aparición de una zona de alta presión en el extremo distal del registro (EEI) y se ajusta de forma que al menos 2 a $3 \mathrm{~cm}$ del extremo distal del catéter se ubiquen en posición subdiafragmática. Sin embargo, esto no siempre es posible debido a la presencia de grandes hernias hiatales o en algunos pacientes con acalasia $(1,9)$. Una forma de verificar la correcta colocación del catéter y asegurarse que éste ha pasado la unión gastroesofágica es pedir al paciente que realice respiraciones profundas, lo que permite identificar más fácilmente la zona de presión distal y el punto de inversión de presión (PIP).

Una vez el catéter se ha posicionado de forma adecuada, se procede al registro de las presiones basales, la evaluación del patrón y vigor contráctil de las degluciones, para lo cual se ha propuesto un protocolo internacional estándar (Tabla 2).

Algunos autores han sugerido que el incremento de la carga de trabajo esofágico en protocolos de manometría puede aumentar la sensibilidad de la investigación. El uso de múltiples degluciones rápidas representa la maniobra provocativa más simple (Tabla 2). Después de la última 
Tabla 2. Protocolo estándar para desarrollar una manometría y pruebas adjuntas durante la manometría esofágica de alta resolución

\begin{tabular}{|c|c|c|}
\hline Acción & Cómo realizarla & Información \\
\hline \multicolumn{3}{|l|}{ Procedimiento estándar } \\
\hline Presión en reposo & $\begin{array}{l}\text { Con el paciente en posición supina o decúbito lateral izquierdo, se le } \\
\text { pide que no realice degluciones por un espacio de } 30 \text { segundos. } \\
\text { Se sugiere realizar respiraciones profundas al final de los } 30 \text { segundos } \\
\text { para detectar el DC. }\end{array}$ & $\begin{array}{l}\text { Detección de las presiones y localización del } \\
\text { EES y EEI. } \\
\text { Permite identificar el PIP. }\end{array}$ \\
\hline Estudio de la peristalsis & $\begin{array}{l}\text { Se realiza dándole al paciente tragos de } 5 \mathrm{~mL} \text { de agua/solución salina } \\
\text { por un total de } 10 \text { degluciones separadas por al menos } 30 \text { segundos. }\end{array}$ & $\begin{array}{l}\text { Vigor y patrón de la función motora } \\
\text { esofágica. }\end{array}$ \\
\hline \multicolumn{3}{|l|}{ Pruebas adjuntas } \\
\hline $\begin{array}{l}\text { Múltiples degluciones } \\
\text { rápidas de bajo volumen }\end{array}$ & $\begin{array}{l}\text { Se realiza dándole al paciente } 5 \text { degluciones rápidas de } 2 \mathrm{~mL} \text { de agua } \\
\text { en } 10 \text { segundos (al menos } 2-3 \text { veces con al menos } 30 \text { segundos de } \\
\text { intervalo). }\end{array}$ & $\begin{array}{l}\text { Se sugiere para evaluar la reserva de la } \\
\text { función motora esofágica y la inhibición del } \\
\text { cuerpo esofágico. }\end{array}$ \\
\hline $\begin{array}{l}\text { Múltiples degluciones } \\
\text { rápidas de alto volumen }\end{array}$ & $\begin{array}{l}\text { Con el paciente en posición de pie, se le da un trago de } 200 \mathrm{~mL} \text { de } \\
\text { agua de manera ininterrumpida. }\end{array}$ & $\begin{array}{l}\text { Se sugiere para evaluar la inhibición del } \\
\text { cuerpo esofágico y cualquier presurización } \\
\text { en/sobre la UGE. }\end{array}$ \\
\hline Tragos viscosos & $\begin{array}{l}\text { Con el paciente en posición supina o de pie, se le da } 5-10 \text { tragos con } 5 \\
\mathrm{~mL} \text { de compota de manzana y otra solución viscosa separados por } 30 \\
\text { segundos. }\end{array}$ & $\begin{array}{l}\text { Intenta detectar el vigor de la peristalsis y } \\
\text { evaluar cualquier presurización en/sobre la } \\
\text { UGE. }\end{array}$ \\
\hline
\end{tabular}

Adaptado de: Savarino E, et al. Dig Liver Dis. 2016;48(10):1124-35. DC: diafragma crural; PIP: punto de inversión de presión; UGE: unión gastroesofágica.

deglución de la serie, se espera una robusta contracción esofágica, la llamada reserva peristáltica. Una respuesta anormal consiste en una inhibición incompleta (cuando se ven fragmentos de contracción durante el período esperado de inhibición) o contracción subóptima (la secuencia posterior a múltiples degluciones rápidas falla para demostrar un aumento de la contracción del músculo liso). Las múltiples degluciones rápidas de bajo volumen evalúan la reserva peristáltica, mientras que las degluciones de grandes volúmenes identifican un aumento de la resistencia al flujo $(4,13)$.

\section{INTERPRETACIÓN DE LA MAR CON BASE EN LOS CRITERIOS DE CHICAGO 3.0}

La MAR demuestra la presión de reposo de los esfínteres y la actividad motora esofágica desencadenada por las degluciones (1). Aunque la mayoría de los análisis se realizan con el software del equipo y son de ayuda, la interpretación final necesita ser desarrollada por un clínico competente y experimentado. El currículo básico de gastroenterología de Estados Unidos (US GI core curriculum) sugiere 2 niveles de entrenamiento para la interpretación de MAR: un nivel básico requerido por todos los gastroenterólogos y un entrenamiento clínico intensivo para los que proveen estudios de MAR a los pacientes (9). Este nivel es requerido debido a la alta prevalencia y carga de salud de los trastornos motores esofágicos (14). A pesar de la anterior recomendación, la mayoría de los programas de formación en gastroenterología no proveen un entrenamiento formal en diagnóstico de motilidad gastrointestinal.

Estudios previos han encontrado que la concordancia en la impresión diagnóstica para MAR esofágica entre el personal en entrenamiento y los médicos en la práctica es subóptima. Un diagnóstico inadecuado impacta adversamente en los resultados de los pacientes y en los costos de salud. El US GI core curriculum recomienda completar 50 estudios de manometría para lograr competencias; sin embargo, un estudio reciente con gastroenterólogos en entrenamiento encontró que $<50 \%$ del personal en entrenamiento logró competencias a un nivel experto luego de evaluar 50 estudios de MAR. Esto sugiere que usar un mínimo volumen de casos para evaluar competencias en MAR esofágica es inadecuado y que parece ser más apropiado establecer puntos de corte para evaluar las competencias de análisis de MAR a través de diferentes niveles $(14,15)$.

La MAR permite diferenciar la presión basal del EEI de la contracción del DC. En condiciones normales, estas 2 zonas de presión deben coincidir, su separación indica la presencia de una hernia hiatal. Asimismo, se puede identificar el PIP, que es el punto donde se da el cambio de presión durante la inspiración de una presión negativa generada por la presión intratorácica a una presión positiva generada por la presión intragástrica; este punto señala la división que 
genera el diafragma entre el tórax y el abdomen (1). Las medidas objetivas de la función esofágica quedan inmediatamente disponibles luego de la realización de la MAR y pueden utilizarse para clasificar las degluciones individuales y generar un diagnóstico de motilidad esofágica (4). Por tanto, cuando se debe hacer el análisis de una MAR es conveniente realizar una interpretación por etapas, como se menciona a continuación.

\section{Presiones basales y función de la UGE}

El estudio se inicia con la evaluación de las presiones de reposo del EES y del EEI (que se identifican como 2 zonas con aumento de presión fácilmente evidenciadas por un cambio de color en la EPT), la morfología de la UGE y si existe o no una hernia hiatal (1). Para lograr esto, es indispensable la colocación correcta de los parámetros de referencia en el software de análisis (Figuras 1 y 2). Hay 2 componentes diferentes que constituyen la UGE: el EEI y el DC. La morfología de la UGE se clasifica en 3 tipos dependiendo del nivel de separación que existe entre la ubicación del EEI y el PIP (que indica la localización del DC) (Figura 3):

- Tipo I: en la cual el EEI y el DC coinciden;

- Tipo II: cuando existe una pequeña separación espacial $(<2 \mathrm{~cm})$ entre el DC y el EEI; $y$

- Tipo III: cuando existe una gran separación espacial $(>2 \mathrm{~cm})$ entre el DC y el EEI $(2)$.

Luego se debe definir si la relajación de la UGE es normal o no durante las degluciones; para esto se evalúa la presión de relajación integrada (IRP), que es una medida de la relajación deglutoria de la UGE (9). Para medir la IRP, el equipo toma 10 segundos a partir del inicio de la deglución (esto es cuando se inicia la relajación del EES). La IRP es la presión promedio más baja en la UGE durante 4 de los 10 segundos mencionados, que pueden ser continuos o discontinuos en una deglución (1) (Figura 4).

Según la nueva clasificación de Chicago, se debe expresar como una mediana (más que una media) de las 10 degluciones, expresada en $\mathrm{mm} \mathrm{Hg}$, con $15 \mathrm{~mm} \mathrm{Hg}$, siendo el límite superior normal para catéteres de diseño Sierra (Given Imaging) (Tabla 3) (9). Un valor superior significa aumento en la resistencia al tránsito del bolo en la UGE y se considera patológica. Cualquier proceso patológico, ya sea mecánico o funcional, que impida el paso en la UGE puede aumentar el IRP. Algunas de estas alteraciones pueden ser la acalasia y condiciones que se comportan como una obstrucción al tracto de salida (neoplasias, estenosis benignas o funduplicatura) (1). Se ha tratado de establecer valores normales de IRP luego de la funduplicatura en pacientes

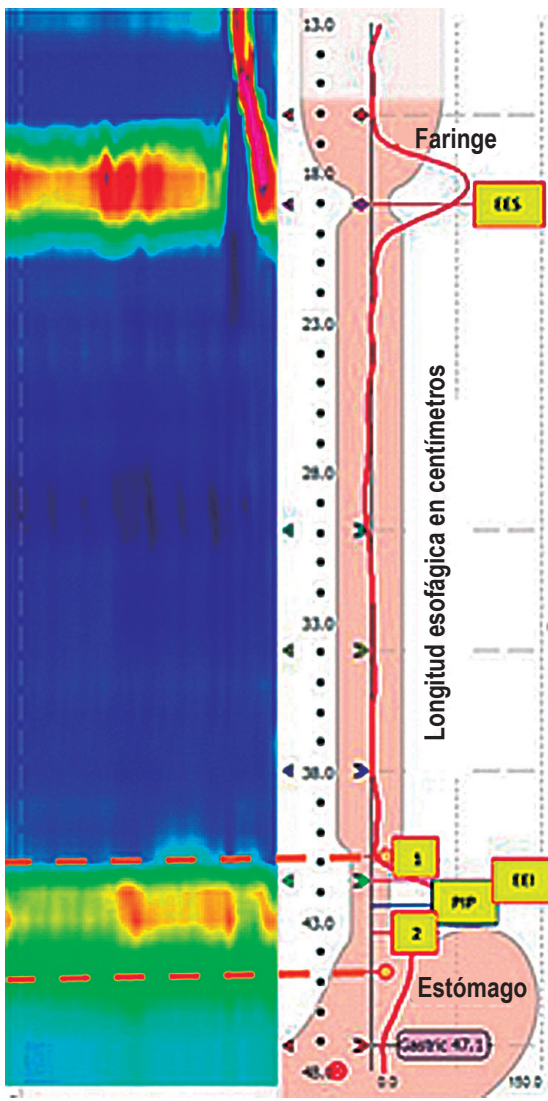

Figura 1. Puntos de referencia del software de MAR. Es necesaria la correcta colocación de los puntos de referencia en el software para lograr un análisis correcto de presiones basales y evaluación de las degluciones. 1: borde superior del EEI; 2: borde inferior del EEI. Los puntos naranjas seguidos por las líneas punteadas delimitan el e-Sleeve para el análisis de la presión residual en la UGE durante las degluciones, por tanto, su ubicación debe abarcar toda la UGE.

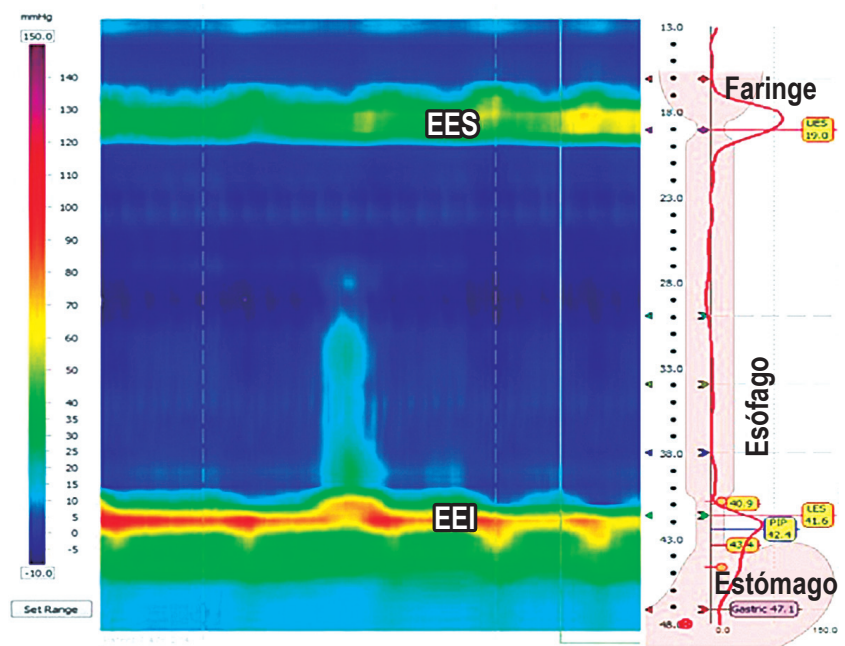

Figura 2. Evidencia de zonas de presión generadas por el EES y el EEI. LES: lower esophageal sphincter; UES: upper esophageal sphincter. 

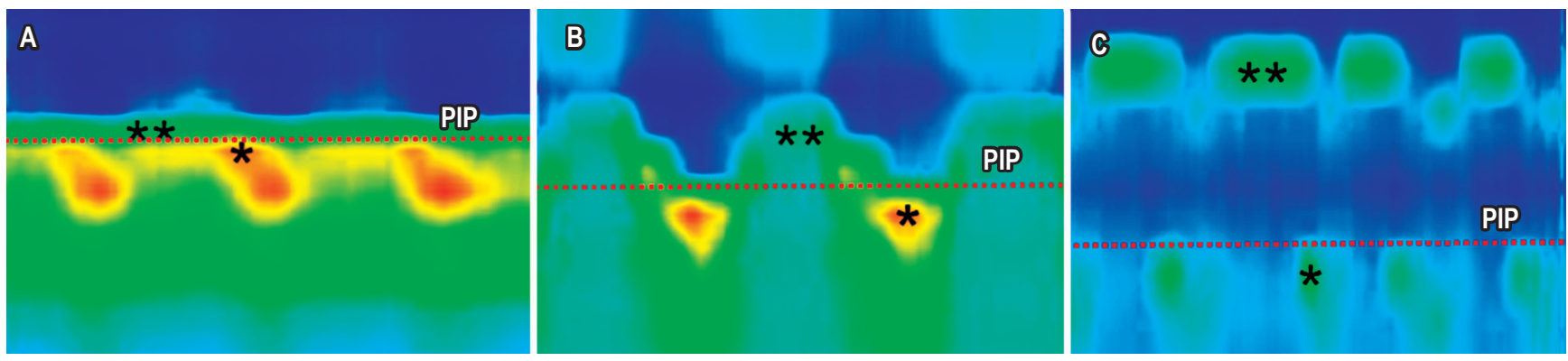

Figura 3. Morfología de la UGE. Se clasifica en 3 tipos dependiendo del nivel de separación que existe entre la ubicación del EEI y el PIP (que indica el punto donde la presión, a través de la UGE durante la inspiración, se invierte de intraaórtica negativa a intragástrica positiva y representa la localización del DC). A. Tipo I, en el cual el EEI y el DC coinciden; B. Tipo II, cuando existe una pequeña separación espacial $(<2 \mathrm{~cm})$ entre el DC y el EEI; C. Tipo III, cuando existe una gran separación espacial $(>2 \mathrm{~cm})$ entre el DC y el EEI. ${ }^{*}$ DC; ${ }^{* *}$ EEI; PIP: línea punteada roja.

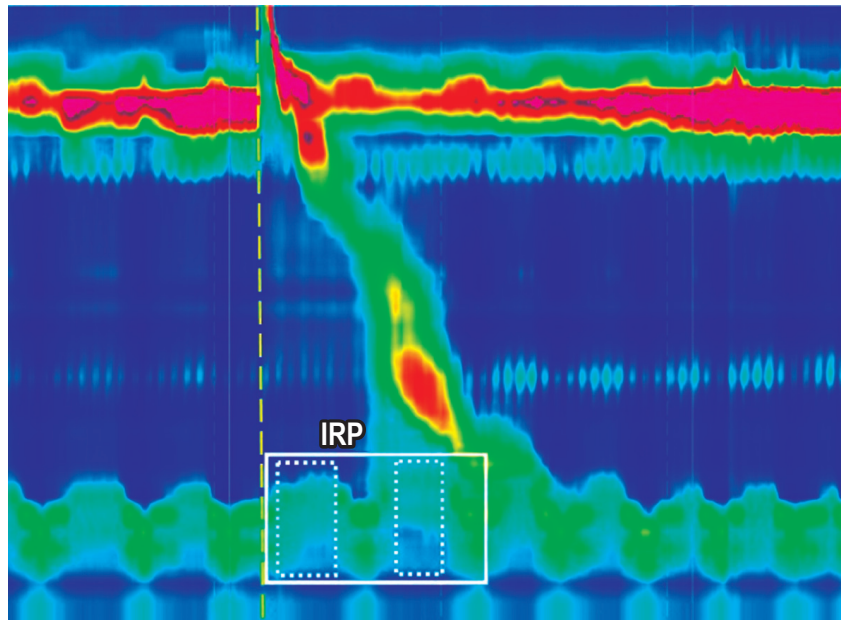

Figura 4. La IRP es la presión promedio más baja a través de la UGE durante 4 segundos (cuadros punteados) continuos o discontinuos dentro de la ventana de 10 segundos (cuadro continuo) en una deglución.

asintomáticos y en un estudio se determinó que el valor del IRP puede ser mayor a lo normal en aquellos con funduplicatura tipo Nissen, pero son similares a los valores normales en sujetos con funduplicatura tipo Toupet (16).

\section{Medidas de EPT para evaluar degluciones individuales}

Las principales medidas en MAR de peristalsis deglutoria usadas en la clasificación de Chicago 3.0 (Figura 5) para evaluar la función contráctil esofágica son la DCI y la DL (Tabla 3).

La DCI es una medida del vigor contráctil (peristáltico) de la contracción esofágica y representa la amplitud $\mathrm{x}$ duración $\mathrm{x}$ largo $(\mathrm{mm} \mathrm{Hg} / \mathrm{s} / \mathrm{cm})$ de la contracción eso-
Tabla 3. Características de contractilidad esofágica en la MAR

\begin{tabular}{ll}
\hline \multicolumn{1}{c}{ Característica } & \multicolumn{1}{c}{ Valor normal } \\
\hline Vigor contráctil (contorno isobárico de los $20 \mathrm{~mm} \mathrm{Hg}$ ) \\
\hline Fallida & $\mathrm{DCl}<100 \mathrm{~mm} \mathrm{Hg} / \mathrm{s} / \mathrm{cm}$ \\
Débil & $\mathrm{DCl}>100 \mathrm{~mm} \mathrm{Hg} / \mathrm{s} / \mathrm{cm}$, pero $<450 \mathrm{~mm} \mathrm{Hg} / \mathrm{s} / \mathrm{cm}$ \\
Inefectiva & $\mathrm{Fallida}$ o débil \\
Normal & $\mathrm{DCl}>450 \mathrm{~mm} \mathrm{Hg} / \mathrm{s} / \mathrm{cm}$, pero $<8000 \mathrm{~mm} \mathrm{Hg} / \mathrm{s} / \mathrm{cm}$ \\
Hipercontráctil & $\mathrm{DCl}>8000 \mathrm{~mm} \mathrm{Hg} / \mathrm{s} / \mathrm{cm}$ \\
\hline Patrón contráctil & \\
\hline Prematura & $\mathrm{DL}<4,5 \mathrm{~s}$ \\
Fragmentada & Pausas $>5 \mathrm{~cm}$ en el contorno isobárico de los \\
& $20 \mathrm{~mm} \mathrm{Hg}$ con $\mathrm{DCl}>450 \mathrm{~mm} \mathrm{Hg/s} / \mathrm{cm}$ \\
Intacta & $\mathrm{No}$ cumple los criterios diagnósticos arriba \\
& mencionados \\
\hline Patrón de presurización intrabolo \\
\hline Presurización & Presurización uniforme de $>30 \mathrm{~mm} \mathrm{Hg}$ \\
panesofágica & extendiéndose desde el EES a la UGE \\
Presurización & Presurización de $>30 \mathrm{~mm} \mathrm{Hg}$ extendiéndose \\
compartimentalizada & desde el frente contráctil a la UGE \\
Presurización de la & Presurización restringida a la zona entre el EEl \\
UGE & y el DC en conjunto con la separación EEI-DC \\
Normal & Sin presurización del bolo $>30 \mathrm{~mm} \mathrm{Hg}$ \\
\hline
\end{tabular}

Adaptado de: Savarino E, et al. Dig Liver Dis. 2016;48(10):1124-35. DCI: integral de contractilidad distal; DL: latencia distal.

fágica en el contorno isobárico de los $20 \mathrm{~mm} \mathrm{Hg}$ a través de las presiones de proximal a distal (4). La DCI es la determinante de degluciones hipercontráctiles (DCI $>8000 \mathrm{~mm} \mathrm{Hg} / \mathrm{s} / \mathrm{cm}$ ), degluciones débiles (DCI $<450$ $\mathrm{mm} \mathrm{Hg} / \mathrm{s} / \mathrm{cm}$ ), peristalsis fallidas (DCI $<100 \mathrm{~mm} \mathrm{Hg} / \mathrm{s}$ / cm) (9). 


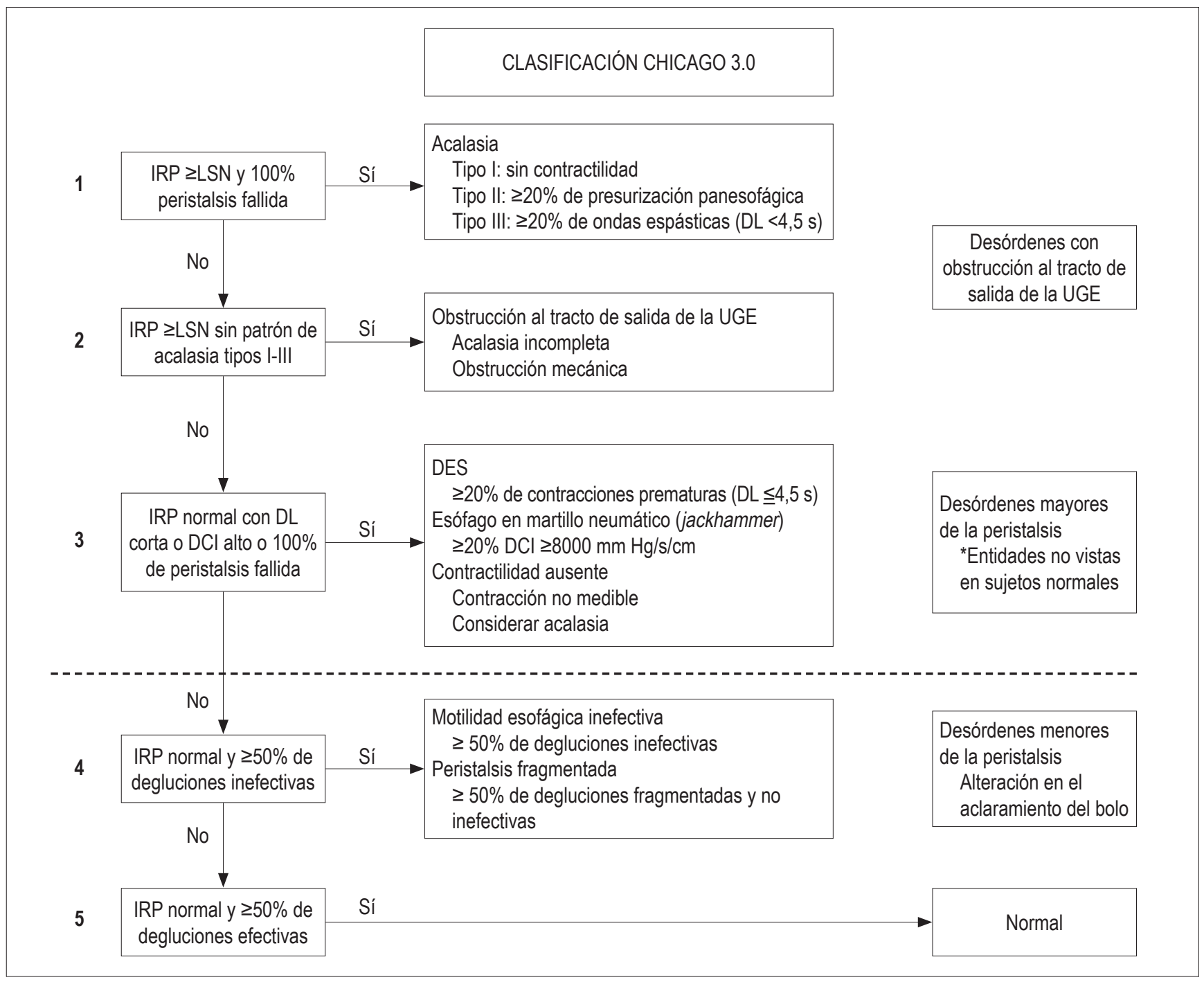

Figura 5. Algoritmo para la interpretación de la MAR esofágica con la clasificación de Chicago 3.0. Tomado de: Kahrilas PJ, et al. Neurogastroenterol Motil. 2015;27(2):160-74. LSN: límite superior de lo normal.

La DL representa el intervalo de tiempo (en segundos) entre la relajación del EES y el punto de inflexión (punto de desaceleración contráctil -CDP-) a lo largo del contorno isobárico de los $30 \mathrm{~mm} \mathrm{Hg}$ donde la velocidad de propagación se enlentece demarcando el esófago tubular de la ampolla frénica. La DL es una medida indirecta de la inhibición deglutiva y, por ende, de la peristalsis normal; y un valor menor de 4,5 segundos define una contracción prematura (9) (Figuras 6 y 7 ).

\section{Pausas peristálticas}

Son brechas espaciales en la contracción peristáltica definidas por examinar la integridad del contorno isobárico de los $20 \mathrm{~mm} \mathrm{Hg}$ desde el EES a la UGE. Las pausas peristálticas se consideran significativas si son $>5 \mathrm{~cm}$ en el eje longitudinal (9).

\section{Patrones de presurización}

Finalmente, se determinan los patrones de presurización anormal. La presurización ocurre cuando el líquido deglutido queda atrapado entre 2 segmentos contráctiles del esófago y se identifica con una banda de presión isobárica vertical. La presión intrabolo anormalmente alta es un signo de alteración en la mecánica del tránsito del bolo y puede ser secundaria a obstrucción del flujo o alteración en la distensibilidad de la pared esofágica. La presurización 


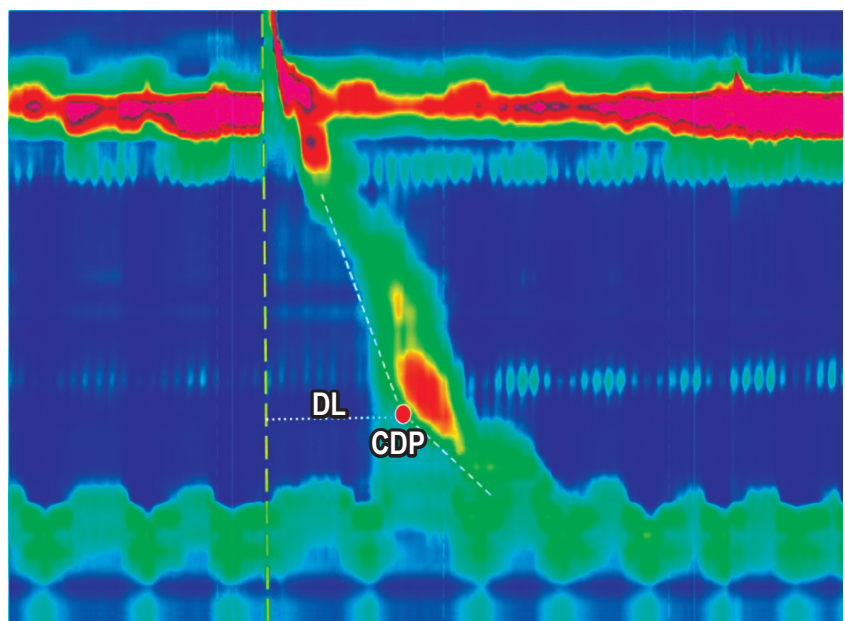

Figura 6. La DL representa el intervalo de tiempo (en segundos) entre la relajación del EES y el CDP (punto rojo). CDP: punto a lo largo del contorno isobárico de los $30 \mathrm{~mm} \mathrm{Hg}$ donde la velocidad de propagación se enlentece demarcando el esófago tubular de la ampolla frénica.

de $>30 \mathrm{~mm} \mathrm{Hg}$ que se extiende desde el EES a la UGE se denomina presurización panesofágica y es la característica definitoria de acalasia tipo II. La presurización compartimentalizada se da entre una contracción peristáltica y la UGE, e indica una obstrucción al flujo de salida.

Pese a desarrollar los estudios de MAR con una técnica consistente y un protocolo estandarizado, se ha demostrado en un hospital de referencia de tercer nivel que los estudios técnicamente imperfectos son comunes y pueden presentarse hasta en un $21 \%$ de los casos. Se consideran estudios

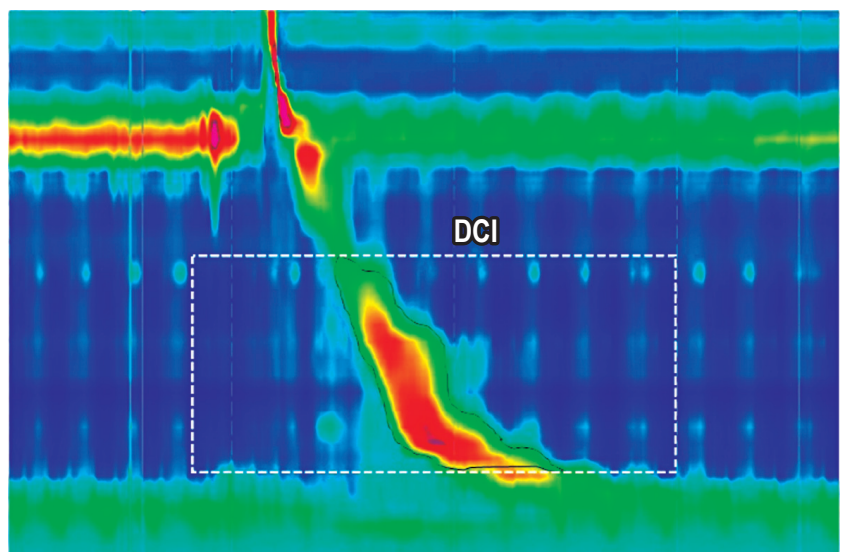

Figura 7. La DCI es una medida del vigor contráctil de la contracción esofágica y representa la amplitud $\mathrm{x}$ duración $\mathrm{x}$ largo $(\mathrm{mm} \mathrm{Hg} / \mathrm{s} / \mathrm{cm})$ de la contracción esofágica en el contorno isobárico de los $20 \mathrm{~mm} \mathrm{Hg}$ (línea negra) a través de las presiones de proximal a distal.

técnicamente imperfectos aquellos que tienen menos de 7 degluciones evaluables, inhabilidad para atravesar la UGE, malfuncionamiento del sensor o compensación térmica alterada, y artefactos misceláneos (vasculares o cardíacos). Sin embargo, pese a las limitaciones técnicas, los estudios pueden aún ser interpretados, especialmente en el contexto asociando los datos endoscópicos y radiográficos (11).

Después de analizar las 10 degluciones, la clasificación de Chicago 3.0 puede ser aplicada para determinar el diagnóstico manométrico del paciente.

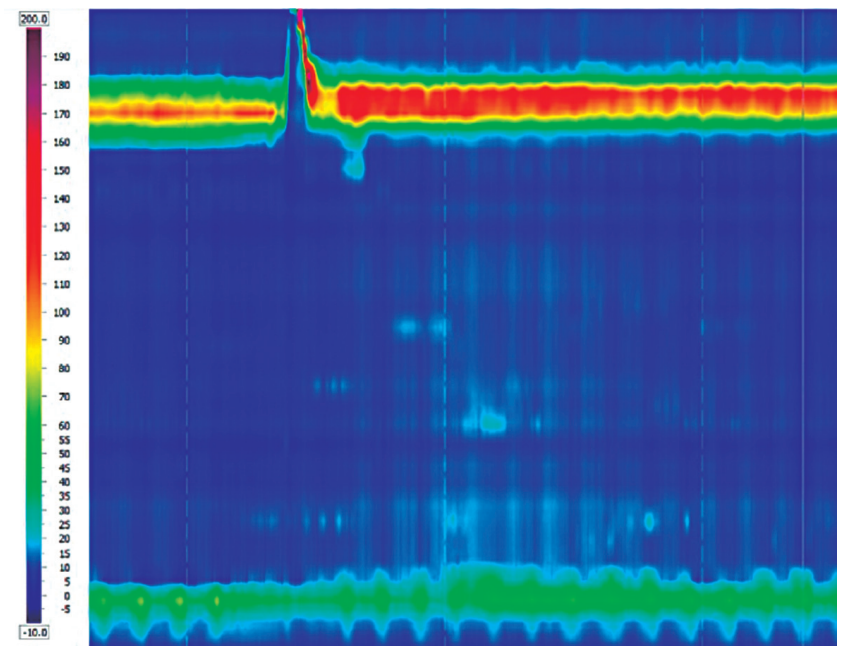

Figura 8. Acalasia tipo I (clásica). Se asocia con la presencia de IRP elevado (>15 mm Hg) y $100 \%$ de degluciones fallidas.

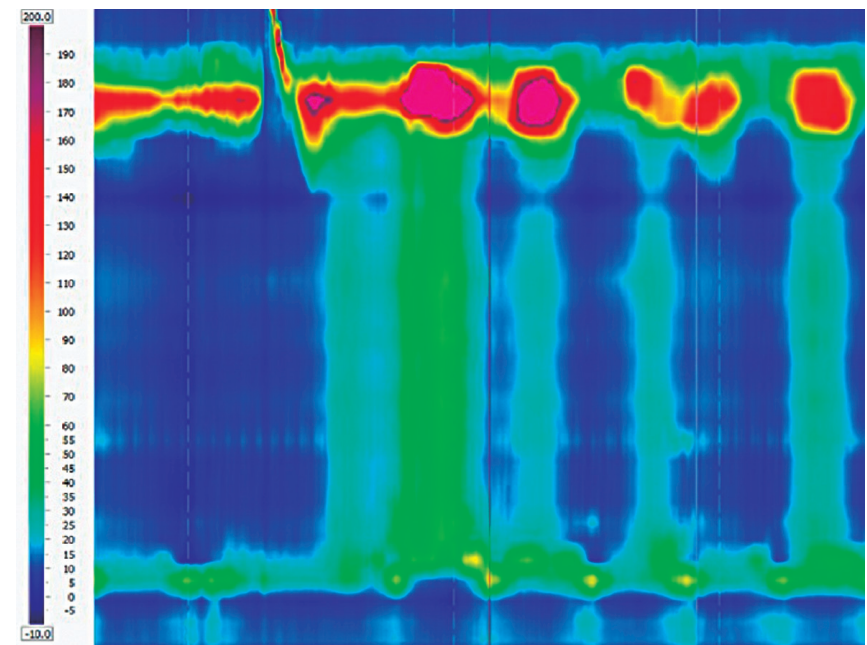

Figura 9. Acalasia tipo II. Se asocia con la presencia de IRP elevado $(>15 \mathrm{~mm} \mathrm{Hg}$ ) y presencia de presurización panesofágica (presurización de $>30 \mathrm{~mm} \mathrm{Hg}$ que se extiende desde el EES a la UGE). 
En el esquema jerárquico de la clasificación de Chicago (Figura 5), la identificación de obstrucción al tracto de salida de la UGE (definida por IRP $>15 \mathrm{~mm} \mathrm{Hg}$ para catéteres de diseño Sierra) es primordial. La presencia o ausencia de esta es el punto de decisión inicial en el algoritmo diagnóstico. Si la obstrucción del tracto de salida de la UGE se acompaña con un $100 \%$ de degluciones fallidas o $20 \%$ de contracciones prematuras, se cumplen criterios para el diagnóstico de acalasia.

La acalasia se subclasifica de acuerdo con el patrón de contractilidad que acompaña a la obstrucción del tracto de salida:

- Tipo I (clásica): se asocia con el $100 \%$ de degluciones fallidas (Figura 8);

- Tipo II (presurización panesofágica): conlleva la mayor posibilidad de éxito al tratamiento (Figura 9);

- Tipo III (espástica): se asocia con una menor tasa de tratamiento exitoso.

Un IRP incrementado asociado con un patrón contráctil que no reúne los criterios para un subtipo de acalasia define una obstrucción del tracto de salida de la UGE. Este es un diagnóstico heterogéneo con diferentes diagnósticos diferenciales que incluyen acalasia temprana o en evolución, obstrucción mecánica, hernia hiatal y artefactos de presión; por lo que evaluaciones adicionales como esofagograma con bario o ultrasonido endoscópico deberían ser considerados antes de alguna intervención terapéutica $(6,9)$.

Si la mediana de IRP es normal, el porcentaje de degluciones prematuras, hipercontráctiles y fallidas puede ser aplicado para identificar un trastorno mayor de la peristalsis, el cual genera patrones que no son observados en sujetos asintomáticos. El DES se define por degluciones prematuras, el esófago hipercontráctil (jackhammer) se define por degluciones hipercontráctiles (Figura 10) y la contractilidad ausente se designa si el $100 \%$ de las degluciones son fallidas $(6,9)$.

Un trastorno menor de la peristalsis es establecido si la mediana de IRP es normal, un trastorno mayor de la peristalsis es excluido y si $\geq 50 \%$ de las degluciones son débiles o fallidas (motilidad esofágica inefectiva) o $\geq 50 \%$ de las degluciones tienen pausas peristálticas grandes (peristalsis fragmentada). Ambos trastornos menores pueden verse en pacientes presentándose con disfagia o síntomas de reflujo. Sin embargo, también pueden verse en controles asintomáticos, por lo que su relevancia clínica permanece no clara. Finalmente, si se descarta un trastorno de motilidad de los descritos previamente $y \geq 50 \%$ de las degluciones son normales, se realiza el diagnóstico de motilidad esofágica normal $(6,9)$.

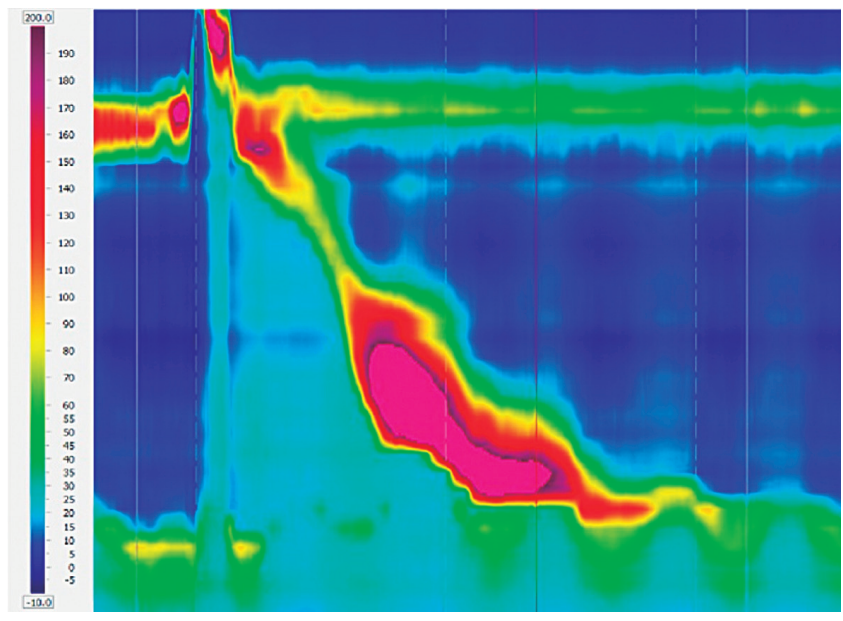

Figura 10. Esófago hipercontráctil (jackhammer). Se define por degluciones hipercontráctiles (DCI $>8000 \mathrm{~mm} \mathrm{Hg} / \mathrm{s} / \mathrm{cm}$ ).

\section{REFERENCIAS}

1. Hani A, Leguízamo AM, Carvajal JJ, et al. Cómo realizar e interpretar una manometría esofágica de alta resolución. Rev Col Gastroenterol. 2015;30(1):74-83.

2. Conklin JL. Evaluation of esophageal motor function with high-resolution manometry. J Neurogastroenterol Motil. 2013;19(3):281-94. https://doi.org/10.5056/ jnm.2013.19.3.281

3. Carlson DA, Pandolfino JE. High-resolution manometry and esophageal pressure topography: filling the gaps of convention manometry. Gastroenterol Clin North Am. 2013;42(1):1-15. https://doi.org/10.1016/j.gtc.2012.11.001

4. Savarino E, de Bortoli N, Bellini M, et al. Practice guidelines on the use of esophageal manometry -A GISMADSIGE-AIGO medical position statement. Dig Liver Dis. 2016;48(10):1124-35. https://doi.org/10.1016/j. dld.2016.06.021

5. Çelebi A. High-resolution manometry versus conventional manometry in diagnosis of patients with nonobstructive dysphagia. Turk J Gastroenterol. 2016;27(6):566-7. https://doi.org/10.5152/tjg.2016.0007

6. Kahrilas PJ, Bredenoord AJ, Fox M, et al. The Chicago Classification of esophageal motility disorders, v3.0. Neurogastroenterol Motil. 2015;27(2):160-74. https:// doi.org/10.1111/nmo.12477

7. Patel A, Cassell B, Sainani N, et al. Comparison of motor diagnoses by Chicago Classification versions 2.0 and 3.0 on esophageal high-resolution manometry. Neurogastroenterol Motil. 2017;29(7):1-8.

8. Monrroy H, Cisternas D, Bilder C, et al. The Chicago Classification 3.0 results in more normal findings and fewer 
hypotensive findings with no difference in other diagnoses. Am J Gastroenterol. 2017;112(4):606-12.

9. Carlson DA, Kahrilas PJ.How to effectively use high-resolution esophageal manometry. Gastroenterology. 2016;151(5):78992. https://doi.org/10.1053/j.gastro.2016.09.024

10. Gyawali CP, Patel A. Esophageal motor function: technical aspects of manometry. Gastrointest Endosc Clin $\mathrm{N}$ Am. 2014;24(4):527-43. https://doi.org/10.1016/j. giec.2014.06.003

11. Roman S, Kahrilas PJ, Boris L, et al. High-resolution manometry studies are frequently imperfect but usually still interpretable. Clin Gastroenterol Hepatol. 2011;9(12):1050-5. https://doi.org/10.1016/j.cgh.2011.08.007

12. Ruiz de León San Juan A, Ciriza de Los Ríos C, Pérez de la Serna Bueno J, et al. Practical aspects of high resolution esophageal manometry. Rev Esp Enferm Dig. 2017;109(2):91-105.
13. Elvevi A, Mauro A, Pugliese D, et al. Usefulness of low- and high-volume multiple rapid swallowing during high-resolution manometry. Dig Liver Dis. 2015;47(2):103-7. https:// doi.org/10.1016/j.dld.2014.10.007

14. Yadlapati R, Keswani RN, Dunbar KB, et al. Benchmarks for the interpretation of esophageal high-resolution manometry. Neurogastroenterol Motil. 2017;29(4):1-7.

15. Yadlapati R, Keswani RN, Ciolino JD, et al. A system to assess the competency for interpretation of esophageal manometry identifies variation in learning curves. Clin Gastroenterol Hepatol. 2017;15(11):1708-14.

16. Weijenborg PW, Savarino E, Kessing BF, et al. Normal values of esophageal motility after antireflux surgery; a study using high-resolution manometry. Neurogastroenterol Motil. 2015;27(7):929-35. 